\title{
SUBSURFACE FLOW IN A STREAM WITH ALTERNATE SANDBARS
}

\author{
Abu Musa Md. Motaher AHMED ${ }^{1}$, Tetsuya SUMI² and Tetsuro TSUJIMOTO \\ ${ }^{1}$ Student Member of JSCE, Postgraduate Student, Dept. of Civil Engineering, Nagoya University \\ (Furo-cho, Chikusa-ku, Nagoya 464-8603, Japan) \\ ${ }^{2}$ Member of JSCE, Dr. of Eng., Research Associate, Dept. of Geo- \& Environmental Engineering, Nagoya University \\ ${ }^{3}$ Member of JSCE, Dr. of Eng., Professor, Dept. of Geo- \& Environmental Engineering, Nagoya University
}

\begin{abstract}
The flow exchange between open channel and alternate sandbar is important for instream ecosystems, water balance and water quality problems investigations. Both experimental and numerical investigations are done to get relationships between surface and subsurface flow in longitudinal and transverse direction. Under steady low flow condition, strong effects of surface flow conditions on subsurface flow structures in the alternate sandbars are recognized through experiments as: (1) Longitudinal flow is dominant, but the longitudinal distribution of subsurface water elevations depend on surface flow conditions. (2) Transverse gradient of subsurface flow varies by surface water discharge and bed shape. Three types of subsurface flow patterns are recognized to define flow exchange between stream and sandbar. Numerical simulations using 2-D theory shows good fit with the experimental results and field data.
\end{abstract}

Key Words: exchange flow, surface flow structure, subsurface flow structure, alternate sandbar, bed shape

\section{INTRODUCTION}

The flow exchange between open channel and alternate sandbar is important for instream ecosystems, water balance and water quality problems. A subsurface flow structure affected by surface flow at the stream-sandbar interface is necessary to estimate and evaluate the exchange flow rates as the direct measurement of flux distribution or total flux through sand bar is not accurate. Areas having high water exchange can be habitat for benthos and hypohreo, which live in substrates near surface water. Small water spaces including backwater along sandbars and temporal ponds also have importance for ecological community. These water spaces are strongly influenced by the subsurface flow structures in the whole sandbar. The pool-riffle-pool sequences formed by alternate sandbars play important role in structuring the subsurface flow paths, especially in the meandering channel.

Some investigators described in brief the subsurface flow structures at the interface with limited physical interpretations. In this paper attempts have been made in order to delineate the subsurface flow structures and the physical parameters sensitive to drive flow exchange between the stream and near stream sand bar for shorter time scale.

\section{PREVIOUS STUDIES}

Interactions between streams and the alluvium on which they flow have been studied widely for various natural processes. Most of the studies are limited to vertical plane. Studies on horizontal interactions are very limited. Kaleris $(1998)^{1)}$ indicated that the factors affecting surfacesubsurface water exchange are hydraulic conductivity of the porous media, hydraulic resistance of the surface water-streambed interface, parameters characterizing the capillary force in porous media, and elevation difference between surface and subsurface water.

Harada \& Takagi $(1996)^{2)}$ discussed on analytical solutions to evaluate minimum discharge at the downstream of a dam in the mountainous region with straight channel and no pool-ripple features. Previous investigations by Wondzell \& Swanson $(1996)^{3)}$, mainly on mountainous stream, show that pattern of subsurface flow changes with the longitudinal bed slope of the stream, stream 
location and influence of secondary channel. Harvey and Bencala (1993) ${ }^{4)}$ demonstrated that topographic variations associated with pool-riffle sequences set up near stream subsurface flow paths.

Kaleris (1998) ${ }^{1)}$ quoted from his previous study that the exchange rate varies in space and time due to fluctuations of water level in the stream and in the aquifer. Larkin \& Sharp (1992) $)^{5}$, Harvey et al. $\left.(1996)^{6}\right)$ and Wroblicky et al. $(1998)^{7}$ reported that the streams with meander bend generate lateral hyporheic zone where flow exchange between stream and subsurface occurs. Wroblicky et al. $(1998)^{7}$ pointed out that the size of the lateral hyporheic zone varies with the hydraulic conductivity of aquifer and stream bed sediment.

\section{OBJECTIVES AND SCOPE OF THE STUDY}

The objectives of the study are to understand the subsurface flow structures in alternate sandbar and its characteristics for various channel flow conditions. In particular, the aim of the study is to determine spatial distributions of subsurface water elevations relative to distributions of surface water elevations for the given flow and bed conditions. These relative distributions are necessary to locate the areas of exchange between surface and subsurface flow and also to calculate the spatial distributions of subsurface fluxes in the sand bar.

Previous studies are mainly based on numerical and field study and most of the studies are concerned mainly with vertical intcractions between stream and underlying porous media. Very few studies are documented to describe stream-sandbar interactions except few tracer studies. In this study, both longitudinal and transverse interactions between stream and sandbar are investigated by flume experiment and numerical simulations.

\section{THEORETICAL CONSIDERATIONS}

Bouwer $(1978)^{8)}$ stated that subsurface flow structure in the vicinity of the stream is threedimensional with vertical velocity component. He also pointed out that when the aquifer thickness is 3 times smaller than the channel width, the flow is horizontal and Dupuit-Forchheimer assumption is then applicable. In the present study, low surface water flow in steady-state condition is considered in order to predict one way interaction between stream and sandbar given that subsurface flow is governed by surface flow conditions and subsurface flow is very small in comparison with surface flow.

According to Dupuit's assumptions, in steady two-dimensional unconfined horizontal flow, $x-$ component of Darcy flux (volumetric flow rate per unit area), $q_{x}$ is given by the Darcy's law:

$$
q_{x}=-k \frac{\partial \phi}{\partial x}
$$

where, $k=$ hydraulic conductivity and $\phi(x, y, z)=$ hydraulic potential. For horizontal bottom impermeable layer with uniform hydraulic conductivity, flux per unit width in horizontal axis, $\bar{q}_{x}$ (i.e. x-component) and Darcy flux, $u$ can be written as:

$\bar{q}_{x}=-k h(x, y) \frac{\partial h(x, y)}{\partial x}, u=-k \frac{\partial h(x, y)}{\partial x}$

where, $h(x, y)=$ subsurface water elevation with respect to impermeable layer.

Under steady-state flow conditions, the continuity equation can be written as:

$$
\frac{\partial \bar{q}_{x}}{\partial x}+\frac{\partial \bar{q}_{y}}{\partial y}=0
$$

Combining Eq.(2) and Eq.(3) and considering isotropic and uniform medium i.e. $k(x, y)$ constant, we get the following simplified equation:

$$
\frac{\partial^{2} h^{2}}{\partial x^{2}}+\frac{\partial^{2} h^{2}}{\partial y^{2}}=0
$$

Eq.(4) is the governing equation provided that the boundary condition implies constant head, for instance, in this case surface water elevations at the edge bounded by the sandbar.

In this study, 2-D characteristics of $h(\mathrm{x}, \mathrm{y})$ and Darcy flux within the permeable layer is discussed.

\section{METHODS}

The investigations are done to get relationships between surface and subsurface flow in terms of water head distributions in longitudinal and transverse directions. The outline of the experiments and numerical simulations are described below:

\section{(1) Experiment}

The experiments are carried out to make clear the relationships between subsurface flow structures and surface flow conditions including bed shape.

A $6 \mathrm{~m}$ long and $50 \mathrm{~cm}$ wide glass-sided horizontal flume is used for experiments. A pump circulated the steady-state discharge, and a stilling basin installed at the upstream provided uniform flow at entrance in the flume. A drainage basin is located at the downstream and the submersible pump is placed into it. The experimental set-up is shown in Fig.1. The water surface slope is adjusted with controls at the downstream, which is also used to reproduce periodic condition around sandbar. Well-graded sand with a median diameter of $2.1 \mathrm{~mm}$ 
is used for preparation of streambed and sandbar. The bed was moulded before each runs and the sandbars are reproduced according to their shape, size and location. The bed elevations $\left(z_{B}\right)$ are given according to the Eq.5 as follows:

$z_{B}=D-(x-\lambda) i_{B}+a \sin \frac{2 \pi(x-0.75 \lambda)}{\lambda} \cos \frac{\pi y}{B}$

where, $i_{B}$ is the longitudinal slope of the flume bed. The average thickness $(D)$ of the permeable layer, sandbar wavelength $(\lambda)$ and width of the flume $(B)$ are taken as $15 \mathrm{~cm}, 300 \mathrm{~cm}$ and $50 \mathrm{~cm}$ respectively. In all cases, bar wavelength is $3 \mathrm{~m}$ and $B / D$ ratio is 3.33. The amplitude $(a)$ of the bars are taken as arbitrary values. The surface of the main channel along thalweg is fixed by uniform spray (used for wood varnishing) ensuring no sediment transport in the flume bed without any effect to permeability. A digital point gauge determined the elevation of the bed and water surface. Twenty piezometer tubes are installed at five lines at $25 \mathrm{~cm}$ interval in $\mathrm{x}$-direction and $5 \mathrm{~cm}$ in $y$-direction (Fig.1) to measure subsurface water potentials (piezometric heads).

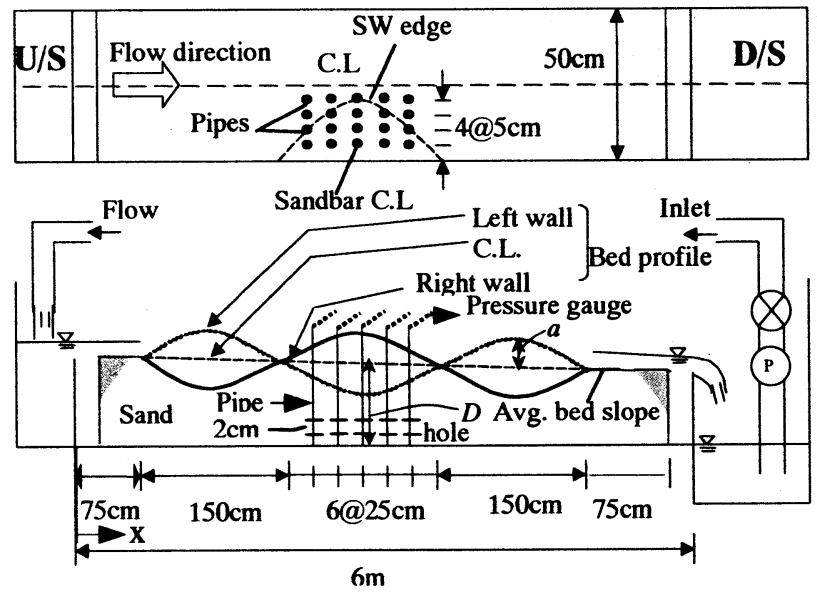

Fig.1 Experimental set-up

\section{(2) Numerical simulations}

Numerical simulations are also performed to compare the experimental results and theory and also as a tool to estimate flux through sandbar and to analyze its trend in detail with introducing geometric information of sandbar and hydraulic index (average hydraulic gradient).

Distribution of surface water elevations at the stream-sandbar interface observed in the experiments is used as the boundary conditions of the subsurface flow model. A 2-D subsurface flow model (FDM, Staggered grid) is used to solve the governing equation (Eq. 4) numerically based on Dupuit-Forchheimer assumptions.

The computation is done in the same hydraulic conditions adopted in the experiment. Spatial distributions of surface water elevation $(H)$ at the boundary and a measured value of hydraulic conductivity $(k=4.96 \mathrm{~cm} / \mathrm{s})$ of sand used in the

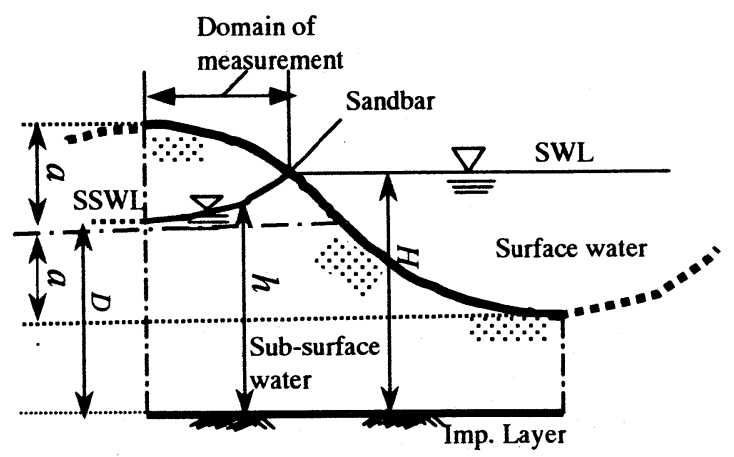

Fig.2 Definition sketch (Transverse view)

experiment are taken for subsurface flow computation. Water exchange between surface and subsurface flow is calculated by using Eq.2. A definition sketch is shown in Fig.2, which provides the physical parameters involved in numerical calculations.

\section{EXPERIMENTAL RUNS}

The response of different amplitudes and bed slopes is studied keeping $B / D$ ratio same for different surface flow conditions. The experiments are conducted for the conditions shown in Table 1.

Table 1 Experimental conditions

\begin{tabular}{|c|c|c|c|c|}
\hline \multicolumn{2}{|c|}{ Bed condition } & \multicolumn{3}{|c|}{ Run No. } \\
\hline $\begin{array}{c}\text { Slope } \\
\left(i_{B}\right)\end{array}$ & $\begin{array}{c}\text { Amplitude } \\
\end{array}$ & \multicolumn{2}{|c|}{ Discharge, $Q$ (Liter/s) } \\
\cline { 2 - 5 } & $\begin{array}{c}1.0 \\
(\text { low })\end{array}$ & $\begin{array}{c}2.0 \\
(\text { medium })\end{array}$ & $\begin{array}{c}3.0 \\
(\text { high })\end{array}$ \\
\hline \multirow{3}{*}{$1 / 100$} & 4.2 & $1 \mathrm{a}-1$ & $1 \mathrm{a}-2$ & $1 \mathrm{a}-3$ \\
\cline { 2 - 5 } & 6.3 & $1 \mathrm{~b}-1$ & $1 \mathrm{~b}-2$ & $1 \mathrm{~b}-3$ \\
\cline { 2 - 5 } & 8.4 & $1 \mathrm{c}-1$ & $1 \mathrm{c}-2$ & $1 \mathrm{c}-3$ \\
\hline \multirow{3}{*}{$1 / 200$} & 4.2 & $2 \mathrm{a}-1$ & $2 \mathrm{a}-2$ & $2 \mathrm{a}-3$ \\
\cline { 2 - 5 } & 6.3 & $2 \mathrm{~b}-1$ & $2 \mathrm{~b}-2$ & $2 \mathrm{~b}-3$ \\
\cline { 2 - 5 } & 8.4 & $2 \mathrm{c}-1$ & $2 \mathrm{c}-2$ & $2 \mathrm{c}-3$ \\
\hline
\end{tabular}

\section{RESULTS AND DISCUSSION}

\section{(1) Experiment}

Subsurface water elevations are plotted at different points within the domain in longitudinal and transverse directions for low, medium and high surface flow conditions. The sensitivity of the subsurface water elevations to the different bed shapes in longitudinal direction is shown in Fig.3 and in transverse direction in Figs.4, 5 and 6. Streambed elevations $\left(z_{b}\right)$ and corresponding surface water elevations $(H)$ are plotted for some typical cases and shown in Fig.7 from which pool-rifflepool sequences can be easily understood. Spatial distributions of subsurface water elevations are shown 

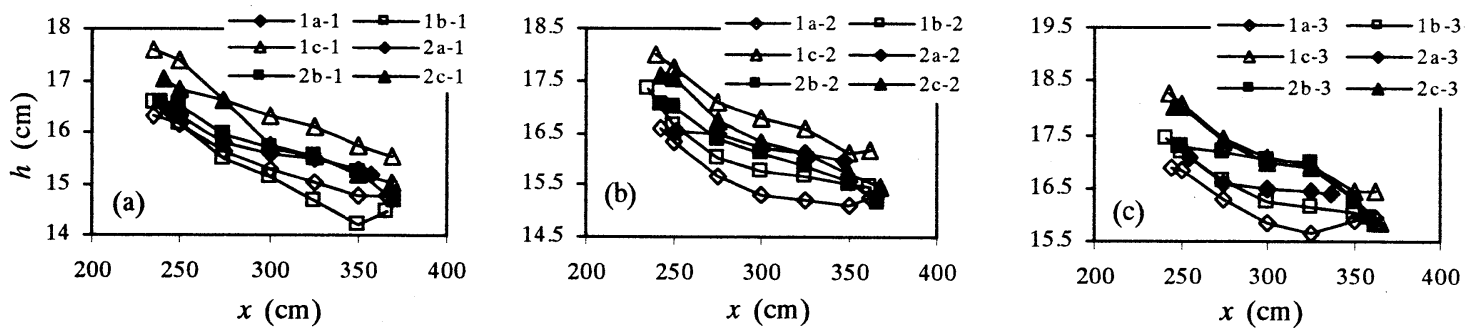

Fig. 3 Subsurface water elevation in $x$-direction at $y=5.0 \mathrm{~cm}$
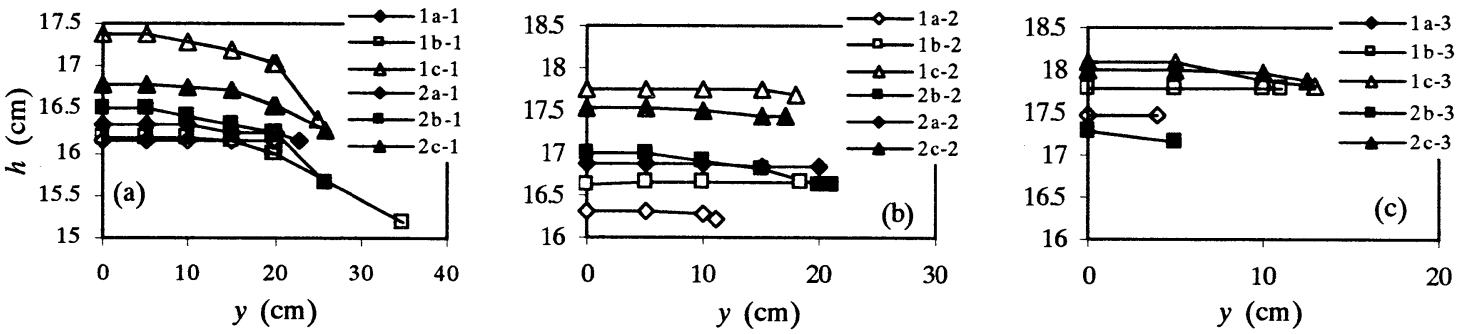

Fig.4 Subsurface water elevation in y-direction at $50 \mathrm{~cm}$ upstream of the sandbar center line $(x=250 \mathrm{~cm})$
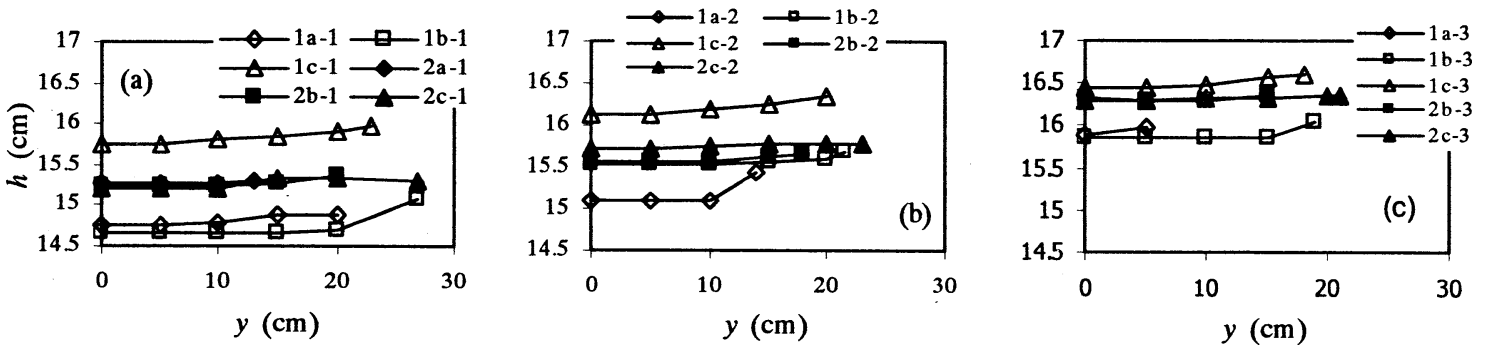

Fig.5 Subsurface water elevation in $y$-direction at $50 \mathrm{~cm}$ downstream of the sandbar center line $(x=350 \mathrm{~cm})$

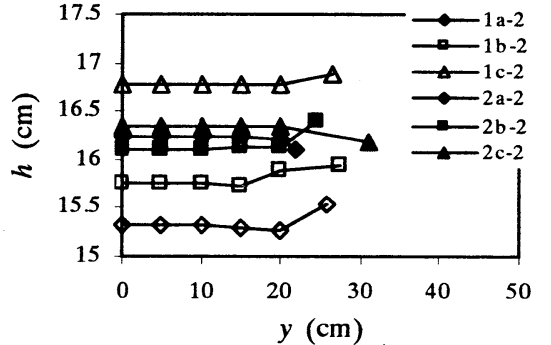

Fig.6 Subsurface water elevation in $\mathrm{x}$ - direction at sandbar center line $(x=300 \mathrm{~cm})$

in Fig.8. These figures are used to discuss the 2-D characteristics of distributions of flux related to surface water distribution and hence possible flow exchange between stream and near stream sandbar.

The analysis can be summarized as follows:

\section{a) Longitudinal trend}

Fig.3 shows that longitudinal profiles of subsurface water level has higher gradient in upstream and downstream region, and lower in middle by the effect of surface water's longitudinal profile with riffle-pool sequence as shown in Fig.7. Especially, upstream region has higher gradient, which shows higher Darcy fluxes in $\mathrm{x}$-direction and higher inflow from surface water. Its trend is weak for lower amplitude cases, and gradient change is gentle in longitudinal direction due to the weaker pool-riffle sequence of surface flow. On the other hand, in case of higher amplitude and higher discharge, downstream region has higher gradient due to higher gradient of surface water extended from down stream with M2 curve. These trends are discussed later with 2-D flow structures including relationship with surface water profile and subsurface transverse flow.

\section{b) Transverse trend}

At the section $50 \mathrm{~cm}$ upstream of the centerline of sand bar ( $x=250 \mathrm{~cm}$, Fig.4), the transverse profiles show concave shape. Its trend is stronger for high amplitude cases (Fig.4(a)). Higher gradient near the surface water edge shows outflow from the sand bar. For high discharge, the similar trend can be seen (Fig.4(c)). For higher amplitude we see high transverse gradient, which is the effect of too high surface water gradient between the two pools (upstream and side pool), so we can expect diverging flow from the upstream end of the sandbar. It indicates high exchange of water in this region (Type I, Fig.10). 

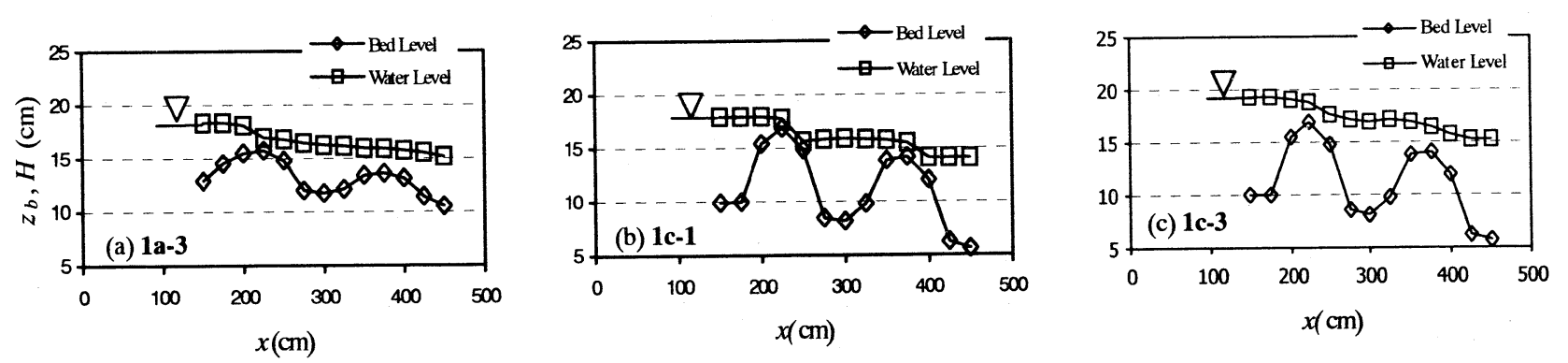

Fig.7 Bed level and surface water elevation in $\mathrm{x}$ - direction

At the section $50 \mathrm{~cm}$ downstream of the center line ( $x=350 \mathrm{~cm}$, Fig.5), we can see small transverse gradient which show the inflow from side pool to the sand bar but it has less sensitivity by the bed shape and discharge. For steep bed slope and high amplitude, transverse gradient appears because of supercritical flow due to higher level of the side pool and hence we can expect converging flow to the downstream end of the sand bar (Type II, Fig.10).

At the centerline (Fig.6), we see the transverse gradient is almost horizontal which indicates no transverse exchange (Type III, Fig.10). In this region longitudinal flow is highly dominant.

\section{(2) Numerical simulations and comparison with} experiment at 2-D viewpoint

The results of numerical simulations are shown as 2-dimensional distributions of subsurface water level and Darcy flux vector within the domain area as shown in Fig.9, which shows similar trend we found in the experiment (Fig.8). The subsurface flow structures found in the experiment and numerical simulations are identical. The fundamental structures of the surface and subsurface flow in the vicinity of the sandbar are schematically shown in Fig.10. This figure shows that 2-D pattern of the subsurface flow at the upstream part of the sandbar is Type I, at the downstream part is Type II and in the middle is Type III. These types of flow pattern occurred due to high surface water gradient (upward trend) at the upstream end, high surface water gradient (downward trend) at the downstream end of the sandbar and low gradient in the side pool.

Comparing the cases as shown in Fig. 8 (or Fig.9), we see that in case 1a-3 the subsurface flow contour lines are almost parallel at the inlet with long and mild upstream edge which allows little inflow and possible outflow beyond the mild edge. This structure is generated by gentle slope of surface water and gradual increase of sandbar width to the down stream. But for the case with same discharge and high amplitude (case 1c-3), the upstream edge has high angle to $\mathrm{x}$-axis at the inlet with small length, and then contour lines have relatively concave shape and their intervals are small.

This indicates inflow part is limited around upstream-end of sandbar, and flow is concentrated.
Outflow can be seen beyond the position where contour lines are perpendicular to the edge line. The contour lines at the downstream also indicate some outflow. This structure is formed due to gradual change in surface water slope. In case $1 \mathrm{c}-1$ the upstream edge line is steep to the wall face and contour lines are bit inclined with edge line, which indicates inflow within the wider area, and contour patterns show strong diverging flow. The downstream contour interval is high which indicates low outflow. But for higher amplitude and high discharge case (1c3 ), outflow is higher due to higher longitudinal gradient by the effect of surface water's gradient as mentioned already.

The inflow and outflow characteristics on budget are shown in Fig.9. Inflows near the upstream end of sandbar contribute most of total inflow flux. Case 1c3 (high $a$ and high $Q$ ) has inflow from side pool because higher gradient of surface water on downstream edge drive subsurface flow partially. Most important characteristic is that most of the flow coming from upstream side goes out to the side pool. This indicates that subsurface flow at upstream part near the riffle is important for water budget and local water movement. In this part, water exchange is high.

It is revealed that the exchange of flow is high in the low flow cases with higher amplitude of the sandbar and less exchange is found for high surface flow with low amplitude.

The subsurface flow structures in a sandbar along Kizu River during low flow condition can be seen in Fig.11. The contour lines show similar trend that we found in the experiment and numerical simulations.

The findings from the numerical simulation based on field data carried out by Wroblicky et al. $\left.(1998)^{7}\right)$ concluded that the extent of the near stream flow systems varied temporally as well and total lateral hyporheic area was reduced during high flow conditions. This phenomenon also satisfies with the findings from the present experimental and numerical study. The above findings were based on the hypotheses that near stream flow systems are generated by geomorphic features such as changes in streambed slope, stream meander bends, and abrupt changes in sediment hydraulic conductivities. In the present study, these hypotheses are proved to be realistic although the hydraulic conductivity through the whole sandbar is assumed as constant. 

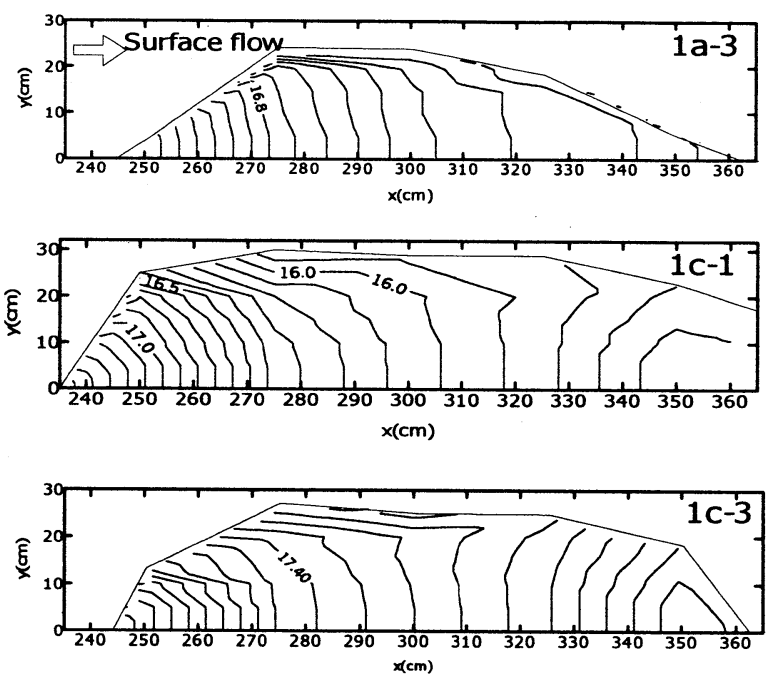

Fig. 8 Spatial distribution of subsurface water elevations (experiment)

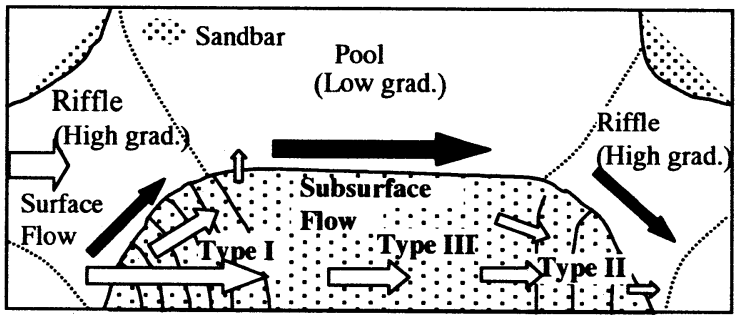

Fig.10 Schematic diagram showing surface and subsurface flow structures at stream-sandbar interface

\section{CONCLUSIONS}

Subsurface flow structures in the sandbar induced by surface flow are investigated through experiments and numerical simulations. Under steady low flow condition, strong effects of surface flow structures on subsurface flow structures in the alternate sandbars are recognized through experiments as:

a) Longitudinal flow is dominant, but the longitudinal distributions of subsurface water elevations depend on surface water conditions.

b) Transverse gradient of subsurface flow varies by surface water flow and bed shape.

Three subsurface flow patterns are recognized to describe the interactions between stream and sandbar.

Numerical simulation using 2-D theory shows good fit with the experimental results and field data.

In this study, horizontal flow characteristics are only discussed. For estimation and evaluation of water exchange quantities with surface water, 3-D analysis is recommended.

\section{REFERENCES}

1) Kaleris, V.: Quantifying the exchange rate between groundwater and small streams, Journal of Hydraulic Research, 36(6), 913-931, 1998.
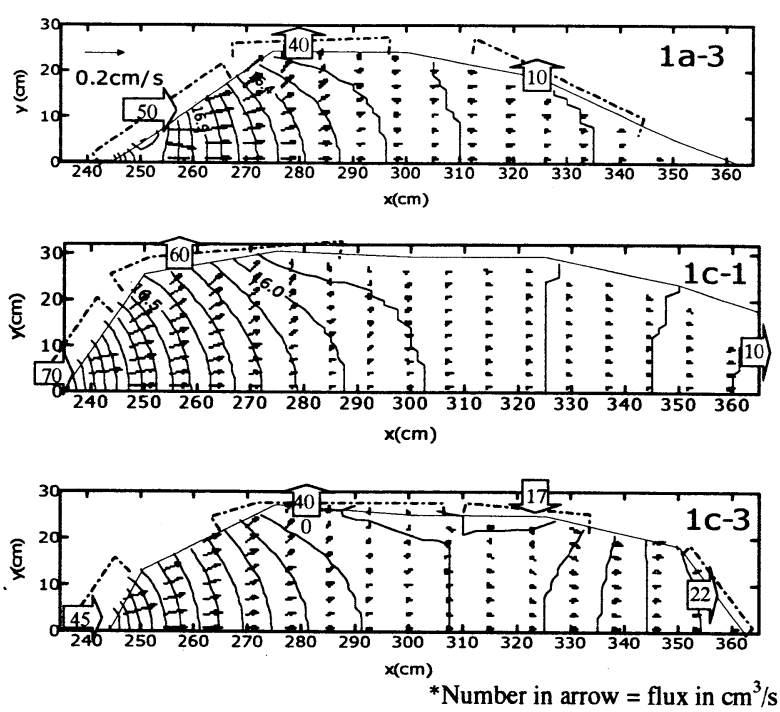

Fig. 9 Spatial distribution of subsurface water elevations and water budget (numerical simulation)

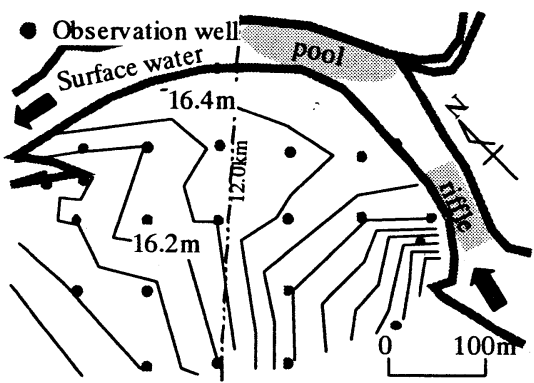

Fig.11 Observed subsurface flow structures in a sandbar along Kizu River (during low flow on Dec. 29, 1999)

2) Harada, M. and Takagi, F.: A Study on Seepage Process of River Water into Sedimentary Layer of Valley and Decrease of Channel Discharge (In Japanese), Journal of Hydraulic, Coastal and Environmental Engineering, 533 (II-34), 21-30, 1996.

3) Wondzell, S.M. and Swanson, F. J.: Seasonal and storm dynamics of the hyporheic zone of a $4^{\text {th }}$-order mountain stream. I: Hydrological processes, $J$. of North Amcrican Benthological Society, 15(1), 3-19, 1996.

4) Harvey, J. W. and Bencala, K. E.: The effect of streambed topography on surface-subsurface water interactions in mountain catchments, Water Resources Research, 29, 8998, 1993.

5) Larkin, R. J. and Sharp, J. M.: On the relationship between river basin geomorphology, aquifer hydraulics, and ground-water flow direction in alluvial aquifers, Geol. Soc. Am. Bull., 104, 1608-1620, 1992.

6) Harvey, J. W., Wagner, B.J. and Bencala, K. E.: Evaluating the reliability of the stream tracer approach to characterize stream-subsurface water exchange, Water Resources Research, 32(8), 2441-2451, 1996.

7) Wroblicky, G. J., Campana, M. E., Valett, H. M. and Dahm, C. N.: Seasonal variation in surface-subsurface water exchange and lateral hyporheic area of two streamaquifer systems, Water Resources Research, 34 (3), 317328, 1998.

8) Bouwer, H.: Groundwater Hydrology, McGraw-Hill, Newyork, 1978. 